\title{
EL TRABAJO EXPERIMENTAL EN FÍSICA COMO ESTRATEGIA DE MOTIVACIÓN. UN TRABAJO DE AÑOS
}

\author{
María Virginia Walz (Universidad Nacional de Entre Ríos)* \\ virginiawalz@bioingenieria.edu.ar \\ Rosa María Weisz (Facultad de Ingeniería. \\ Universidad Nacional de Entre Ríos*** \\ romaweisz@yahoo.com.ar \\ Roberto Luis Albarenque (Facultad de Ciencias de la Educación. \\ Universidad Nacional de Entre Ríos) $)^{* * *}$ \\ roalbaren@yahoo.com.ar
}

Recibido: 31/07/2012 Aceptado: 10/11/2012

\section{Resumen}

La predisposición a realizar actividades está en estrecha relación con la motivación. En la práctica socioeducativa de los educandos, el desarrollo de las esferas motivacional y cognitiva es de vital importancia en la formación del alumno. Las motivaciones que tiendan a que el individuo permanezca en actividad por sí mismo (motivaciones intrínsecas) son las que producen los mejores resultados en los procesos y aprendizaje. Cuando hablamos de motivación en la práctica docente, casi nunca se la relaciona con el docente y sólo se le pide que sea capaz de estimular a sus alumnos. ¿Pero un docente desmotivado, puede motivar a sus alumnos?

Dentro de este contexto, el trabajo que presentamos aspira a acercar a docentes y alumnos de primaria y secundaria algunas experiencias didácticas de física por medio de las cuales los alumnos puedan construir su propio conocimiento. Ellos realizan las experiencias propuestas e intentan diseñar nuevos experimentos como respuesta a situaciones problemáticas y desafíos planteados, con la ayuda y la guía del docente.

* Master en Ingeniería Biomédica. Facultad de Ingeniería de la Universidad Nacional de Entre Ríos.

Bioingeniera. Facultad de Ingeniería de la Universidad Nacional de Entre Ríos.

** Especialista en Ingeniería Clínica. Universidad Tecnológica Nacional (convenio UTN-Favaloro). Experta Universitaria en Robótica y Visión por Computador. Universidad de Alicante (España). Bioingeniera. Facultad de Ingeniería de la Universidad Nacional de Entre Ríos.

*** Analista Programador de Sistemas. Universidad Autónoma de Entre Ríos. 
Trabajamos con materiales comunes y accesibles que permitan demostrar, con montajes sencillos, fenómenos físicos cotidianos y que sean fácilmente reproducibles por los alumnos en sus hogares así como por los docentes en sus aulas.

\section{Palabras claves}

Motivación docente-alumno - experimentación - interactividad - física.

\section{Abstract}

The predisposition to perform activities is in close relation with one's motivation. In the socio-educational practice of the students, the development of the motivational and cognitive areas is of vital importance in to the learners' growth. This motivation that moves individual learners to engage in an activity by himself/herself is that type of motivation (intrinsic motivation) that produces the best results in the teaching and learning processes. When we talk about motivation in the teaching practice, this is seldom related to the teacher and it is only referred to in relation to the ability to stimulate the students. However, can a demotivated teacher motivate his/her students?

Within this context, the work we present aims to offer teachers and students of primary and secondary education some didactic physics experiences by means of which the students can build their own knowledge. The participating learners carry out the suggested tasks and experiences and try to design new experiments in response to problematic situations and challenges presented, with the help and guidance of teachers.

We work with common and accessible materials to demonstrate, with simple configurations, everyday physical phenomena that are easily reproducible by the students in their homes as well as teachers in their classrooms.

\section{Key words}

Teacher-student motivation - experimentation - interactivity - physics.

\section{Introducción}

La motivación humana no es una variable observable. Lo que motiva al alumno a ser activo y participativo en las actividades que el docente ha programado para desarrollar en el aula es algo difícil de saber, además de conocer que no todos los alumnos responden de la misma manera. La voluntad de aprender esta íntimamente ligada a la motivación, que no sólo tiene que ver con la seguridad que siente el alumno en la clase con su profesor y compañeros, sino también con la confianza y estabilidad familiar. La motivación tiene muchas aristas pero, en la práctica educativa, transparenta el vínculo entre el proceso de aprender y el de enseñar.

La motivación es un aspecto fundamental de la personalidad, que en el campo de la psicología, junto a las necesidades humanas, constituye el núcleo central para la persona [1]. Entonces deberíamos preguntarnos ¿qué es la motivación? y para ello tomaremos la definición que realiza González Serra en 
su libro Psicología de la motivación que dice: "Por motivación se entiende la compleja integración de procesos psíquicos que efectúa la regulación inductora del comportamiento, pues determina la dirección (hacia el objeto-meta buscado o el objeto evitado), la intensidad y el sentido (de aproximación o evitación) del comportamiento" (González Serra, 2008: 52). O dicho en otras palabras: "La motivación despierta, inicia, mantiene, fortalece o debilita la intensidad del comportamiento y pone fin al mismo, una vez lograda la meta que el sujeto persigue" (González Serra, 2008: 52).

El estudio de las teorías psicológicas de la motivación se desarrolla en torno a dos grandes posturas, según se trate desde la estructura cognitiva humana 0 desde la conducta humana frente a un estímulo. Estas representaciones definen dos clases de motivaciones. Una de las categorías, conocida como motivación extrínseca, hace referencia al logro de los diferentes niveles de satisfacción personal, como expresa Romo [2]. Por otro lado, la motivación intrínseca, según Bruner [3], tiene que ver con tres aspectos primarios: cómo atrae al alumno la situación problemática que el docente le presenta, qué lo estimula a desarrollar sus habilidades individuales y colectivas para permanecer activo en esa situación planteada, y cómo actúa según la demanda del problema expuesto.

En este sentido, entendemos altamente relevante reflexionar desde nuestro rol de docente sobre qué condimentos trabajar para mejorar la motivación de los alumnos, pero también sobre qué aspectos de nuestra misión educativa innovar para estar más motivados y así transmitir entusiasmo en la práctica, teniendo en claro que ciertas motivaciones son sólo del alumno como individuo y no están en el quehacer del docente, mientras que tanto la metodología que utilice el profesor como su relación con el alumno son de suma importancia. Por ejemplo: ¿estamos creando clases interesantes? ¿Estamos incorporando sus preocupaciones e intereses en nuestra planificación? ¿Los contenidos curriculares pueden extrapolarse su vida cotidiana? o visto desde otro plano, preguntarnos ¿qué aspectos de nuestras clases lo desmotivan? ¿Serán muy teóricas? ¿No "bajamos" las teorías a la vida cotidiana? ¿No les visualizamos y concretizamos el fenómeno estudiado?, entre muchísimas otros planteamientos. Es claro que, en la práctica docente no se busca diferenciar un tipo de motivación de otro, pues además continuamente se mezclan y complementan fusionándose en una sola conducta, sino poder diferenciar tareas y acciones que nos permitan alcanzar nuevos aprendizajes a partir de conocer el grupo de alumnos, tanto desde el punto de vista humano personal como desde la competencia individual y colectiva y el desarrollo de habilidades.

Con este propósito y junto al de tender un puente entre la visión universitaria y la realidad presente en el ámbito escolar y apoyar el trabajo de sus docentes, desde la Facultad de Ingeniería de la UNER estamos trabajando con nueve escuelas de educación primaria y secundaria, públicas y privadas, de la ciudad de Paraná. A partir de la construcción de un escenario compartido no formal, 
nuestro trabajo pretende acercar a los alumnos experiencias científicas prácticas de física óptica, eléctrica y de sonido. Las mismas se plantearán de modo que sean simples, de fácil entendimiento y reproducción por los alumnos en sus hogares así como por los docentes en sus aulas. Orientadas a representar acontecimientos físicos cotidianos que, por sobretodo, les permita anclar los conocimientos adquiridos en las aulas y vislumbrar el carácter empírico de la ciencia.

Con todo esto, intentamos estimular el aprendizaje por descubrimiento. Citando a Gellón (2005), pretendemos "ayudar al alumno a tomar conciencia de sus propias ideas preexistentes, dándole oportunidad para confrontarlas, debatirlas, afianzarlas o usarlas como andamiaje para llegar a ideas más sofisticadas" [4]. Queremos desmitificar la idea de que la ciencia es aburrida estimulando el placer y el goce de nuestros estudiantes.

A continuación deseamos exponer detalladamente nuestro trabajo, que desde hace casi una década estamos desarrollando, así como también el resultado que estamos consiguiendo.

\section{Marco teórico. Antecedentes}

Nuestra tarea se inscribe en una línea de trabajo en Divulgación y Popularización de la Ciencia que lleva más de una década. En el año 1999, el equipo interdisciplinario del "Museo Interactivo de Ciencias Puerto Ciencia" de la UNER desarrolló el proyecto de Investigación "Eficacia de la Interactividad en la enseñanza de las ciencias" [5], en el que una de las acciones llevadas a cabo fue la vinculación con dos instituciones sitas en la ciudad de Paraná: El Teatro Municipal 3 de Febrero -a través de la Secretaría de Cultura y Deporte de la comuna local- y el Museo Puerto Ciencia. Es así, que en el año 2004, bajo el título "El Teatro de Visitas", comienzan a programarse visitas a nuestro teatro destinadas especialmente a docentes y alumnos de EGB 3, Polimodal y universitarios; aunque también, pretendía acercar la cotidianidad de la ciencia a un mayor número de personas. Si bien este Programa tenía como centro a la física y a las matemáticas, no excluía a las otras ciencias que hacen al vasto mundo teatral creando un canal más entre Arte y Ciencia [6]. En tal sentido, se realizaron encuentros guiados recorriendo el teatro, con el propósito de ampliar los segmentos que asisten al mismo, no solamente desde el punto de vista del espectáculo, sino desde otros aspectos muy amplios. La particularidad de estos encuentros era sociabilizar conceptos brindando explicaciones sobre los fenómenos ópticos, de iluminación y sus aplicaciones; descripciones geométricas y matemáticas y sus ventajas; principios de transmisión del sonido y la acústica del teatro. Todos desde una mirada científica a partir de una simple observación selectiva de los objetos y formas del teatro [7] conjugada con la visión del docente en búsqueda de actuar en forma extrínseca sobre la estimulación del alumno. 
La propuesta se basó en el objetivo que persigue el Método Didáctico Alternativo de "Descubrimiento guiado" que procura incrementar el conocimiento de los estudiantes acerca de un tema determinado estimulando su curiosidad (motivación intrínseca) con el objeto de inducirlos a desarrollar estrategias generalizadas orientadas a que aprendan a aprender procedimientos para descubrir conocimientos en otras situaciones similares o diferentes [8]. Una docente expresa: "En esta guía, se busca entusiasmar a los alumnos en la exploración e incentivarlos en el desarrollo del sentido de investigación y de la actividad intelectual, haciéndolo a través de desafíos, de planteos" [9].

Aunque existieron algunos factores que condicionaron el completo logro de las visitas (por ejemplo, que no se pudieran realizar algunas experiencias porque ciertos sectores del teatro se encontraban momentáneamente cerrados al público por mantenimiento) la motivación por parte del docente fue esencial, ya que se trataron temas que previamente se desarrollaron en forma teórica en el aula, pero sin embargo muchos no dominaban, al igual que cierto lenguaje específico. La meta era conseguir que los estudiantes quieran aprender, aunque sabiendo que esto era muy difícil, pues cada uno de ellos tenía intereses diferentes; pero fueron atrapados por las experiencias planteadas.

En el mismo año, se realizó la disertación sobre el programa educativo "La ciencia en el teatro" dentro de las actividades organizadas para el desarrollo de la Semana de la Ciencia y la Tecnología. Asimismo, en esta instancia desde el Museo se realizaron diversos talleres dirigidos a público en general, entre los que nos encontrábamos dedicadas a temas de óptica con desarrollo de experiencias de gran impacto visual al trabajar con luces de colores y láseres didácticos. El Diario de Paraná [10] subraya la actividad señalando una de las premisas que fundan la creación del Museo Puerto Ciencia y que se repite en cada una de nuestras acciones: difundir aspectos del conocimiento - en particular científico - a través de experiencias de física, matemática y química, entre otras, sustentadas en la idea que postula la importancia de ver y manipular para incorporar datos y conceptos.

En la ciudad de Gualeguaychú, Entre Ríos, en octubre del 2006, vivimos una experiencia altamente satisfactoria. En esta carnavalesca localidad tuvo lugar el 8vo. Simposio de Investigadores de Educación de la Física (SIEF 8). Dentro del marco de este evento, y simultáneamente con el mismo, se llevaron a cabo dos interesantes actividades: la exposición del Museo Itinerante Puerto Ciencia y el taller Experiencias cotidianas de Óptica que complementan la práctica curricular formal del ciclo escolar [11]. Este taller no tenía un carácter convencional, en el sentido de que no se le había asignado un horario en la planificación de actividades del evento. Sino que, por el contrario, durante todo el transcurso del simposio estaba ubicado en un lugar de tránsito abierto al público que asistía al mismo. La idea era experimentar, "jugar con lo que había" y descubrir fenómenos ópticos. Esto es, se acercaban quienes así lo desea- 
ban. Los organizadores del simposio invitaron a las instituciones educativas de la zona, primaria, secundaria y técnicas. Las mesas de experimentación se colmaron de alumnos y docentes; muchos se acercaban por curiosidad, otros para conocer y los más ansiosos buscaron probar y experimentar. Tomado del Museo Interactivo de Ciencias, el lema era: "Prohibido no tocar" y así fue que cada visitante, o en grupo reducido, - ahora en su mayoría escolares - recorrían el stand armando y construyendo libremente la experimentación planteada así como debatiendo y concluyendo los desafíos propuestos, que podían incluir desde armar un periscopio con prismas de reflexión total hasta construir un microscopio compuesto.

Inesperadamente, nuestro asombro por el gran entusiasmo y motivación que percibimos en los asistentes, fue inmenso. También es muy interesante destacar que varios docentes se acercaron a consultar sobre la construcción y desarrollo de equipamiento para la enseñanza de la ciencia en sus aulas.

Entre 2006-2008, surge como iniciativa un trabajo de extensión desde el interior de la asignatura Óptica Geométrica y Física de la Facultad de Ciencia y Tecnología de la Universidad Autónoma de Entre Ríos (FCyT-UADER) llevado a cabo por las docentes Virginia Walz y Rosa Blasón y los estudiantes que cursaban en ese momento la asignatura mencionada.

Cuando reflexionamos sobre nuestras prácticas escolares surgen ideas y conclusiones que deben ser ordenadas y analizadas con el objetivo de optimizar el proceso de enseñanza aprendizaje. Tal como venimos contando, al explorar nuestro trabajo y trayectoria como docentes de Física, la motivación y el compromiso del docente, en primer lugar, como del alumno son fundamentales para el trabajo y desarrollo educativo en el aula. Los docentes tenemos muchas responsabilidades que no sólo tienen que ver con impartirles conocimiento, sino que también con orientarlos, ayudarlos a reforzar su propia confianza y autoestima y, quizás lo mas difícil de lograr, incentivarlos a aprender y valorar la ciencia. El saber pedagógicamente cómo mantener al grupo de estudiantes interesados en las actividades y tareas propuestas por el docente es importantísimo para un mejor logro del aprendizaje, pero también se debe ser consciente de que no todos los alumnos responden igual. El nacimiento del proyecto arriba mencionado estuvo en estrecha relación con esto; es decir, con el actuar, desde nuestra posición de docente, desde el plano extrínseco de la motivación. El propósito de este proyecto es plantearnos que el aprendizaje de las ciencias debería constituir una tarea fascinante que despierte el interés de los alumnos por penetrar en los secretos de ese mundo cambiante del que forman parte y a partir del cual construyen su conocimiento. Sin embargo, la articulación de los trabajos experimentales con la actividad del aula no es tarea sencilla, se intenta disminuir esa dificultad y lograr en los alumnos una actitud positiva frente al estudio de las ciencias. Cabe además mencionar que muchas veces la falta de lugares adecuados para el desarrollo de trabajos de laboratorio, 
recursos didácticos, recursos para insumos, materiales, etc., no sólo provocan desánimo para buscar alternativas sino que, hasta parecen influir en la calidad del conocimiento científico.

En el año 2008 se visitaron dos instituciones educativas con mucho éxito, previa autorización y charla con autoridades y docentes y se recibieron solicitudes de visitas de otras, no sólo de la ciudad de Paraná. En esta ocasión el diario UNO de Paraná entrevistó extensamente a las docentes responsables del proyecto [12]. En el artículo bajo el nombre Incentivan el aprendizaje de Física en el Nivel Medio, se recalcan los términos "construcción del conocimiento" y "significatividad del aprendizaje", así como "realimentación entre alumno y docente". En la Figura 1 podemos ver a los chicos trabajando en los distintos talleres.
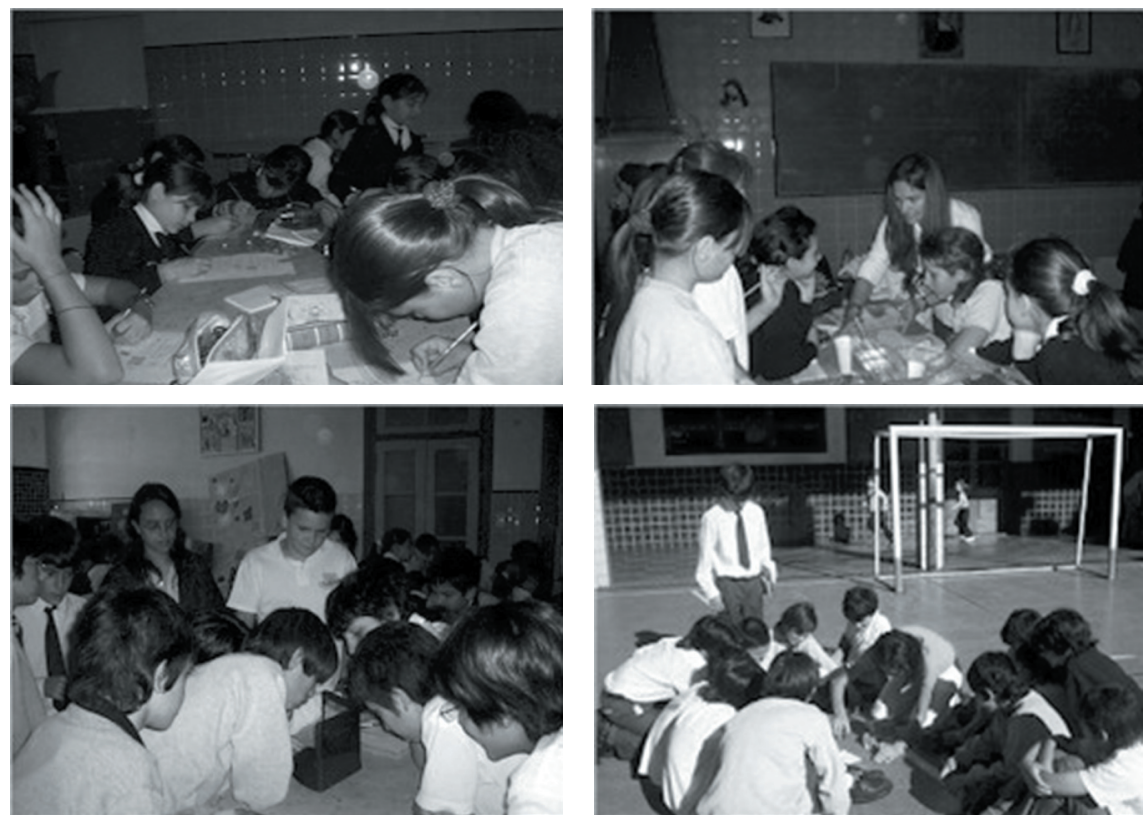

Figura 1: Alumnos desarrollando diferentes actividades experimentales

Por otra parte, el éxito de estas visitas se conoció al relevar los datos de las encuestas realizadas a los alumnos al final de cada taller. También surgió del análisis posterior de las observaciones de los docentes a cargo de las experiencias, conjugadas con los comentarios de los docentes a cargo de los cursos. Teniendo en cuenta que se visitaron dos instituciones educativas y que en cada encuentro participaron más de 40 alumnos, totalizamos unas 80 encuestas. Las visitas en general resultaron positivas, los chicos afirmaban 
en su mayoría que conocían los fenómenos, pero no los habían investigado o no sintieron la necesidad de cuestionarse el por qué de ellos y el cómo funcionaban ciertos instrumentos. En cambio, otros expresaban que era la primera vez que observaban situaciones de estas características, como por ejemplo, la trayectoria de la luz, algo tan cotidiano.

En la Figura 2 se muestra parte de la encuesta realizada a los alumnos y los resultados obtenidos en una escuela.

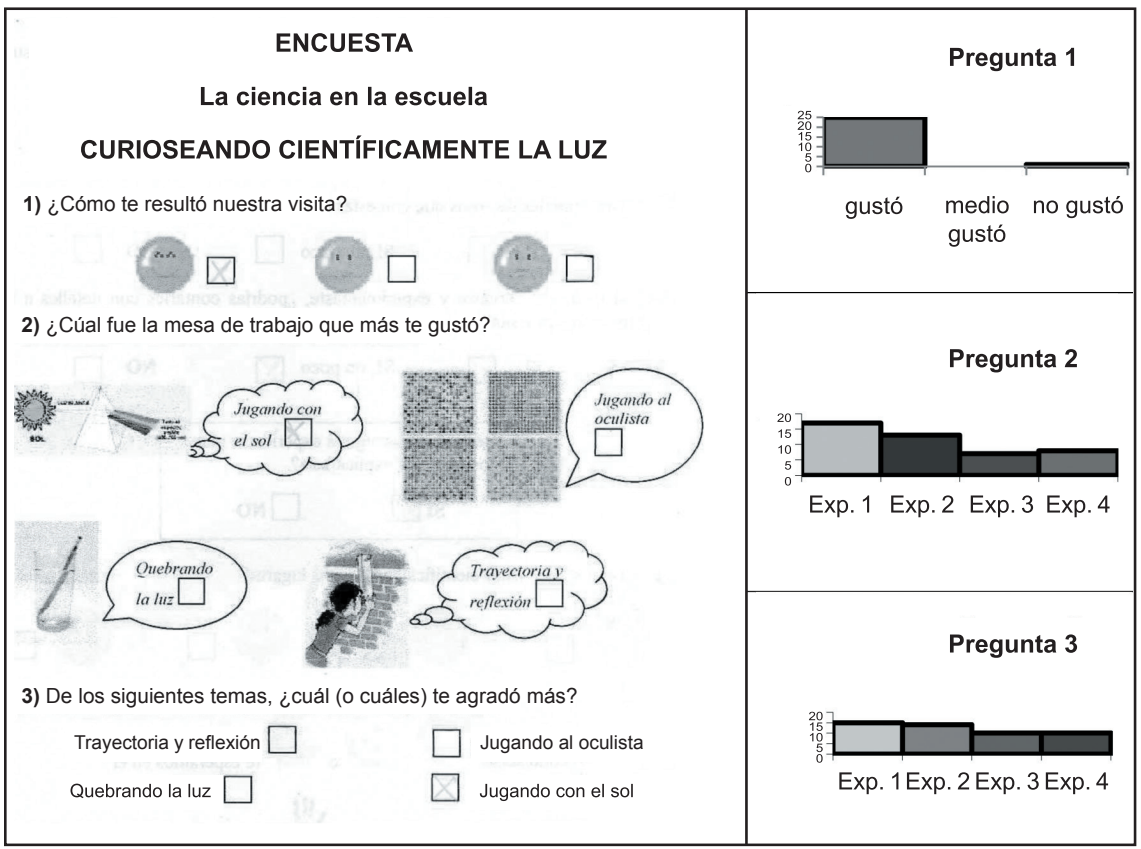

Figura 2: Parte de la encuesta realizada a los alumnos.

Obsérvese la motivación provocada en cada experiencia

En la Figura 3 se observan algunas experiencias planteadas en el cuadernillo que a cada alumno se le entregó con el objetivo de guiarlo a descubrir el concepto físico a través de ellas.

En junio de 2007 participamos, como siempre, en la Semana de la Ciencia y la Tecnología (que tuvo lugar en el Museo Interactivo de Ciencias "Puerto Ciencia"). En esta oportunidad, presentamos una extensa variedad de experiencias, situaciones problemáticas y desafíos; siempre todos con el mismo objetivo: buscar generar interrogantes, despertar el interés, motivar hacia la investigación y el descubrimiento en las ciencias experimentales. 

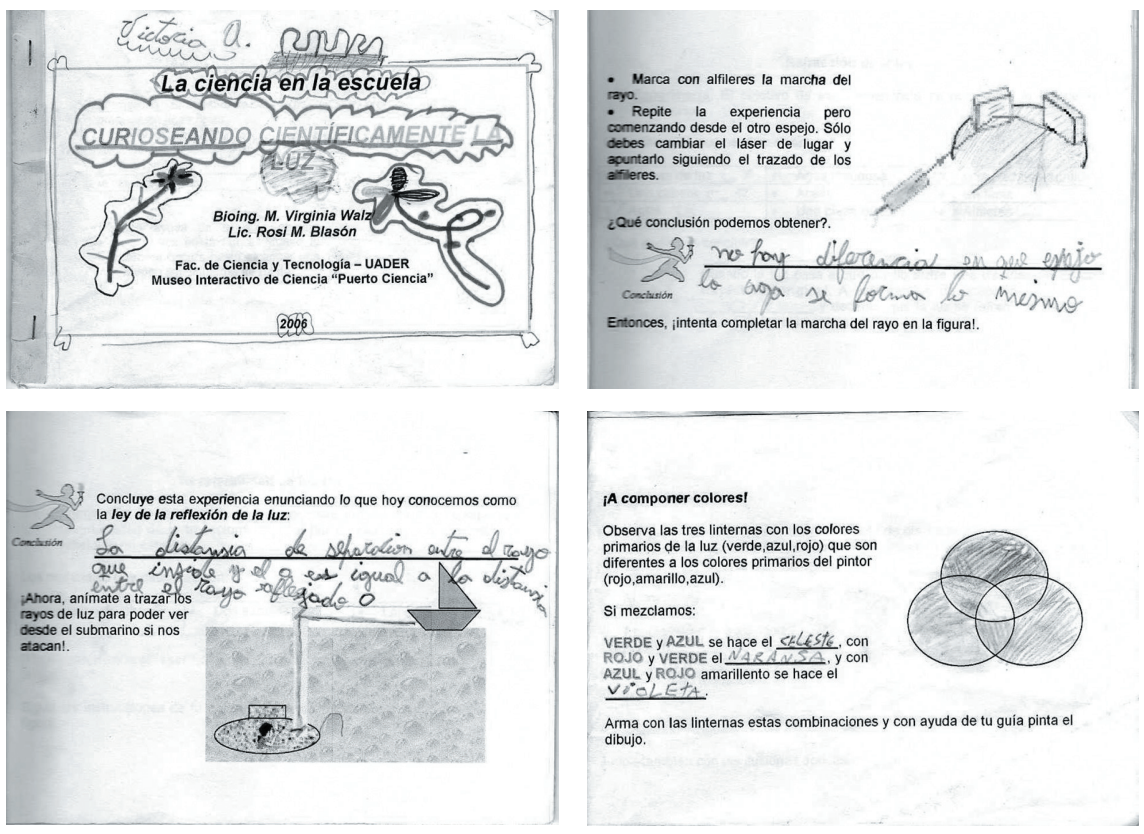

Figura 3: Descripción de los alumnos de experiencias planteadas

El trabajo docente consiste tanto en planificar actividades de enseñanza adecuadas a cada objeto de estudio que motiven a los estudiantes en su aprendizaje, proporcionen la información necesaria y promuevan mecanismos de control y de regulación de este proceso, como en construir un entorno de aprendizaje que fomente un ambiente de clase y valores tendientes a facilitar la comunicación de las ideas y las formas de trabajo, el intercambio de puntos de vista, el respeto, su confrontación y la elaboración de propuestas consensuadas, logrando de esta manera mejorar la calidad educativa.

Nuestra tarea nos ha llevado a desarrollar una percepción en el espacio educativo acerca de cómo ir adecuando una clase en función de la participación de los alumnos, cuándo detenernos en algún punto, cuándo seguir avanzando, cuándo llamar la atención sobre alguna cuestión. ¿Pero qué sucede cuando ninguna de estas acciones hace mella en el estudiante y sentimos y observamos que miran para otro lado? Tal vez lo primero que se nos viene a la mente es que no les interesa nada.

Sabemos que la gran preocupación de los docentes de Física y en general de las Ciencias Naturales es cómo atrapar el mundo real y reabrirlo en el aula haciendo que el alumno sea partícipe de este redescubrimiento y por las razones antes mencionadas vemos que los trabajos de laboratorio, experimentales, 
son un elemento importante sobre el cual obrar para lograr el mejoramiento de calidad educativa. En concordancia con la voluminosa bibliografía de muchísimos colegas e investigadores educativos, nacionales e internacionales, el aprendizaje resulta más productivo en calidad y cantidad cuando hay motivación intrínseca, porque se mantiene por sí mismo, sin factores externos.

En nuestro afán de integrar a todos los estudiantes, buscamos alternativas para despertar su interés y no siempre resultan exitosas. Las actitudes de indiferencia de los alumnos no suceden en todos los ámbitos académicos, cada grupo es diferente, cada situación es diferente, incluso para un mismo ámbito académico se dan situaciones de grupos más participativos que otros. Si entendemos que enseñar es diseñar actividades sociales con el propósito deliberado de que alguien aprenda algo, hemos de admitir que posiblemente no siempre resulte adecuado para todos los participantes. Todos hemos vivido la angustiosa situación de enseñar cosas que los alumnos no aprenden y lo lamentable es que a este estado le sigue con mucha frecuencia la resignación. Y esos mismos alumnos han vivido también con irritación, paciencia y apatía la situación inversa de ver cómo alguien les enseña cosas que ellos no estaban en disposición de aprender.

Cómo no pensar en que el alumno se pueda desmotivar ya sea por la personalidad del docente, su comportamiento autoritario, por la ausencia de material didáctico, por un inadecuado método de enseñanza. Asumir la motivación desde la perspectiva del alumno sería una mirada acotada de las posibles causas de la falta de interés, o de la disociación del espacio enseñanza-aprendizaje, también sería conveniente asumir la motivación desde el plano del docente. Hay muchos factores que podrían afectar la motivación del docente tanto desde su órbita personal como otros vinculados con la institución en donde desarrolla sus actividades.

Al acto educativo habría que verlo en una relación bidireccional en la que el docente tendría que contemplar las cosas qué pueden ser útiles para que el vector del que habla González Serra se vea favorecido con una conducta activa en la clase por parte del alumno y que a su vez produzca una realimentación hacia el docente para estimularlo a realizar una clase más participativa y más potencializadora del aprendizaje. Así mismo, diseñar situaciones en donde el alumno tenga un protagonismo ayuda a disparar el estímulo para conocer nuevas situaciones enriquecedoras. Es necesario tener en cuenta que todo esto debe contar con la energía suficiente para superar la inercia de no aprender, la cual es diferente en cada persona y puede ser motivada por el entusiasmo que el docente transmite como en un acto de "contagio" de las "ganas" de aprender y que puede debilitarse con sólo utilizar en el discurso docente un lenguaje que no lo apasione, con lo que probablemente no entusiasme. 


\section{Acerca del desarrollo del proyecto actual y su metodología}

Nos proponemos continuar con esta metodología de trabajo, contagiando las ganas de aprender, acercándonos a escuelas, primaria y secundaria, públicas y privadas, previo encuentro con los docentes a cargo de los grupos, y plantear con ellos diversas actividades prácticas, que ilustren los fenómenos que estudiaron o estudiarán en clases. Buscamos que dichas actividades experimentales estén orientadas al desarrollo de una nueva mirada y renovación de perspectivas escolares a través de la educación no formal por lo cual la producción científica logra significativa presencia en la realidad educativa.

Haciendo uso de recursos didácticos no convencionales, pero con materiales fáciles de conseguir en nuestra vida cotidiana. La idea es trabajar en talleres para propiciar la participación activa, y promover en los asistentes, un cambio de los arquetipos asociados a lo que entienden como conocimiento científico. Citando a Izquierdo (2007): buscamos contribuir a la formación de alumnos competentes en el pensamiento científico, creativo (caracterizados por lo que saben hacer y que no reproducen conductas estereotipadas) [13].

Mediante la reflexión y la interacción, tratamos de contribuir a la popularización de la ciencia, haciendo que la asimilación de sus principios resulte más efectiva. Como dice Gellón (2005), queremos que los chicos aprendan a "hacer ciencia como los científicos" [4].

En cuanto a los docentes, esperamos acercarles una estrategia metodológica alternativa, que contribuya a mejorar la enseñanza de carácter experimental así como brindarles un material que los ayude a incorporar nuevas experiencias en sus clases y por otro tanto complementar las existentes, ayudando a mantener "en alto" el estado de motivación que requiere el escenario áulico.

Prospectivamente, el proyecto apunta a contribuir a que a los alumnos les quede una nueva mirada sobre el fenómeno físico y la naturaleza del conocimiento científico. Respecto a los docentes, les quedaría el material didáctico y experimental cedido por el grupo, que podrían utilizar para trabajos futuros en sus aulas. En ambos, pretendemos sembrar la inquietud por seguir desarrollando actividades empíricas con los materiales que tengan a su alcance.

Aquí, nos parece interesante continuar este relato describiendo, aunque de manera resumida, algunos de los objetivos planteados en nuestro proyecto: - constituir una estrategia didáctica definida, precisa e interesante que haga comprensible la física para que ésta pueda ser divertida y a la vez atractiva; - fortalecer y enriquecer las destrezas de enseñanza de la física con innovaciones metodológicas; contribuir a la enseñanza significativa de la física óptica, eléctrica y del sonido y a la formación científica del alumno y del docente; - potenciar y consolidar el conocimiento científico en los alumnos; - demostrar a través de experiencias sencillas realizadas por alumnos y docentes en el aula, fenómenos físicos en la vida cotidiana y aplicaciones corrientes de leyes y principios; - promover la creatividad y el desarrollo de instrumentos sencillos 
de trabajo para ser utilizados en el aula por docentes y alumnos, además lograr recrear los fenómenos en sus casas.

En las visitas a los establecimientos educativos, las actividades previstas se llevarán a cabo conjuntamente con el docente a cargo y los alumnos. Aunque cada módulo cuenta con un texto informativo, los docentes son los encargados de motivar e informar a los estudiantes. Esta actividad también es realizada por los alumnos integrantes del proyecto. Nuestro proyecto cuenta con la participación de alumnos avanzados en Bioingeniería (UNER), en el Profesorado en Física y en la Licenciatura en Criminalística (UADER).

En una primera instancia se concretaron reuniones previas con el docente a cargo del curso, con el fin de seleccionar algunos ejes temáticos básicos del área de física óptica, eléctrica y de sonido que forman parte de la currícula escolar, principalmente de la escuela secundaria, y que se advierten asiduamente en la vida cotidiana, alrededor de los cuales se construyen conceptos, se determinan propiedades y se plantean situaciones problemáticas diversas a partir de experiencias, en su mayoría, de tipo cualitativa. Con el objetivo de hacer que la ciencia sea más amigable y menos aburrida, elaboramos experiencias y guías con estas características relacionando conceptos científicos.

Luego, se construirán kits de experimentación para cada eje temático. Algunos de ellos son: naturaleza de la luz, leyes ópticas, óptica en el ser humano, descomposición de la luz, circuitos eléctricos en serie y paralelo con y sin interruptores, cajas acústicas para caracterizar cualitativamente los principales parámetros físicos de la onda sonora: frecuencia, intensidad, sonidos graves y agudos, débiles y fuertes, así como parámetros de sonoridad: timbre, altura, tono. Se elaborará un material escrito, que llamamos cuadernillo, compuesto por las diferentes experiencias diagramadas con el objetivo de guiar al estudiante a descubrir y formalizar el concepto físico a través de ellas. Se incluirán desafíos y planteos deseando activar en los alumnos la curiosidad intelectual dando oportunidad a que piensen, se activen cognitiva y afectivamente y generen el feed-back necesario para constatar el nivel de comprensión que van consiguiendo. Por ejemplo, en la actividad que hace referencia a las leyes de reflexión y refracción, el desafío consiste en lograr que ellos construyan la trayectoria de la luz en un submarino, además de investigar sobre los diseños actuales. En cuanto a situaciones problemáticas de física eléctrica, el desafío esta orientado a investigar y analizar algunas de las conexiones eléctricas de su casa e intentar reproducirlas; mientras que desde las ondas sonoras los desafíos parecen ser mayores al proponer la construcción de un modelo simple de oído humano, la interferencia de ondas sonoras o "hacer bailar" a esferas de telgopor.

Cada curso visitado se organiza en grupos de trabajo, uno por kit, guiados por un coordinador, donde cada alumno tiene a su disposición el cuadernillo.

En todo este contexto es necesaria una continua comunicación (personal y virtual) entre los profesores y los docentes donde se intercambien ideas, con- 
tenidos curriculares, actividades áulicas, disponibilidad de horarios, espacios para el desarrollo de las experiencias y todo lo que sea concerniente al buen adelanto del proyecto y del proceso enseñanza-aprendizaje.

Para lograr la comunicación virtual con los docentes y mantenernos próximos a ellos es que utilizaremos un recurso digital, implementando un espacio virtual de articulación mediante una plataforma que nos brindará las herramientas necesarias para tal fin. De esta manera se define un punto de reunión virtual para que entre todos los participantes encontremos un lugar común para obtener material didáctico relacionado a las experiencias de laboratorio. Este recurso le permite a los docentes descargar archivos en el momento que los requieran utilizándolo como una biblioteca virtual.

Otra posibilidad que nos brinda la plataforma es la de intercambiar opiniones mediante uso de foros. Esta es una potente herramienta de comunicación asíncrona que nos permitirá enriquecer los contenidos del espacio virtual mediante el aporte de los diferentes docentes en cada temática propuesta y desarrollar el proceso de aprendizaje de forma grupal, validando la acumulación de experiencias individuales y colectivas así como los diferentes puntos de vista ante determinados planteamientos.

Por otro lado, la mensajería interna con utilización de correo nos facilitará la comunicación de ida y vuelta con los docentes acerca de sus inquietudes como también brindarnos un canal de envío de información o material que ellos crean de interés para sus pares.

También, para complementar el recurso didáctico publicado en la plataforma y así potenciar el fin de los mismos, podremos colocar videos instructivos sobre la instrumentación de las experiencias. Y como divulgación de nuestras actividades en las diferentes instituciones educativas crearemos un espacio de difusión en la misma plataforma con fotografías y videos de nuestro itinerario.

Creemos que el efecto multiplicador de la plataforma nos permitirá llegar más allá de nuestra capacidad física para visitar diferentes establecimientos lejanos a nuestro lugar de residencia.

Para un posterior análisis del impacto del proyecto y del grado de cumplimiento de sus objetivos, se realizarán, entre otras, encuestas a los participantes.

\section{Resultados esperados y perspectivas a futuro.}

Con este proyecto, aspiramos a acercar la universidad a las escuelas, contribuyendo a ampliar el canal de vinculación entre las mismas.

Esperamos que los docentes de las distintas escuelas participantes continúen usando los materiales que les dejemos y que éstos les sirvan para crear y construir nuevas experiencias. Deseamos que la experiencia pueda servir de puntapié inicial para qué otros docentes que no participaron de la misma se interesen por esta alternativa metodológica. Anhelamos que se propague la idea de que se pueden reproducir experiencias sencillas, usando materiales 
alternativos, no convencionales, de bajo costo y de fácil acceso. Creemos que el uso del campus virtual de la UNER puede contribuir a que continúe el vínculo entre los docentes que participaron de los talleres junto a sus alumnos y los profesores universitarios a cargo de los mismos, favoreciendo y fortaleciendo la comunicación y el intercambio de ideas.

Pretendemos continuar sembrando entre los estudiantes: la inquietud por experimentar, probar, cuestionar, analizar y entre los docentes: por un lado, la conciencia de que sin motivación no será posible conseguir resultados que nos satisfagan como docentes y por el otro, la sabiduría de conseguir un escenario experimental agradable y no improvisado que permita generar conflictos cognitivos que despierten la curiosidad e interés del alumno y orientar las tareas para mantener el interés por aprender.

\section{Referencias Bibliográficas}

- González Serra, D. J. (2008). Psicología de la motivación. La Habana: Editorial Ciencias Médicas.

- Romo, M. (1997). Psicología de la creatividad. Barcelona: Paidós.

- Bruner, J.S. (1988). Desarrollo cognitivo y educación. Morata (Selección de textos por Jesús Palacios).

- Gellón, G., Rosenvasser Free, E., Furman, M. \& Golombek, D., (2005). La ciencia en el Aula. Buenos Aires: Paidós.

- Proyecto "Eficacia de la interactividad en la enseñanza de las ciencias". Estudio sobre resultados en los visitantes del museo. Facultad de Ingeniería, UNER, 1999-2004. Código: 07/F055. Resolución probatoria: № 078 CS UNER (15/5/2000).

- Berardi, O., Gandulfo, M. I. \& Walz M. V. (2005). La matemática y su presencia cotidiana. Libro de Resúmenes del VII Simposio de Educación en Matemática. Sociedad Argentina de Educación Matemática. Chivilcoy. Buenos Aires.

- Gandulfo, M. I., Berardi, O. \& Walz, M. V. (2005). La ciencia desde un teatro. Revista Novedades Educativas, 17(179), 68-72.

- Good, Th. \& Brophy, Y. (1996). Psicología educativa. México: McGraw-Hill Interamericana.

- Berardi, O., Gandulfo, M.I. \& WALZ, M.V. (2004). Entrevistas en medios de difusión (radios, televisión y diarios) y publicación en un sitio de internet oficial relacionadas con actividades en la UNER a los autores sobre el programa "La ciencia en el teatro" dentro de las actividades organizadas en la "Semana de la Ciencia y la Tecnología". Teatro Municipal "3 de Febrero". Paraná. E. Ríos.

- $\quad$ Gandulfo, M. I. \& WALZ, M. V. (2004). La ciencia ahora también va al teatro. El Diario. Paraná. Entre Ríos.

- Blasón, R. M. \& WALZ, M. V. (2007). Experiencias cotidianas de óptica en la enseñanza media. Revista Novedades Educativas, 18(199), 80-81.

- Walz, M. V. \& Blasón, R. M. (2008). Incentivan el aprendizaje de Física en el Nivel Medio. Diario UNO. Paraná. Entre Ríos.

- Izquierdo, M. (2007). Enseñar ciencias, una nueva ciencia. Enseñanza de las ciencias sociales, 6, 125-138. 